\title{
Violencia externa en el trabajo en el sector servicios. Consideraciones generales, notificación y protocolos de actuación
}

\author{
External violence at work in the service sector general considerations. Notifica- \\ tion and action protocol
}

María Luisa Rodríguez de la Pinta, María Maestre Naranjo, Jenry Ricardo Borda Olivas

Servicio de Prevención de Riesgos Laborales. Hospital Universitario Puerta de Hierro. Majadahonda (Madrid). España.

\author{
Correspondencia: \\ María Luisa Rodríguez de la Pinta \\ Servicio de Prevención de Riesgos Laborales \\ Hospital Universitario Puerta de Hierro \\ $\mathrm{C} /$ Joaquín Rodrigo 2 \\ 28222 Majadahonda (Madrid). España \\ Tfno.: +34911917415 \\ E-mail: mlrodriguez.hpth@salud.madrid.org
}

Resumen

La violencia en el lugar de trabajo es un fenómeno emergente que afecta a todos los sectores y categorías laborales. Su prevención y manejo exige una actuación coordinada de todas las instituciones implicadas.

Partiendo de la normativa legal existente, se crea la necesidad de poner en marcha las medidas preventivas dirigidas a eliminar o minimizar el riesgo, para conseguir este objetivo se deben elaborar procedimientos de trabajo que establezcan las actuaciones a seguir ante situaciones de violencia externa, estos procedimientos son de aplicación a todos los trabajadores y tienen que ser difundidos a todos los estamentos de la empresa.

La información y formación de los trabajadores en relación con este tema es otra de las medidas preventivas que hay que poner en marcha, para que estos adquieran las habilidades necesarias con el fin de evitar la violencia externa.

Para conocer la dimensión exacta del problema es preciso disponer de los datos referidos a las situaciones de violencia externa que suceden en nuestra organización, por lo que es imprescindible elaborar un plan de notificación y registro.

Palabras clave: Violencia externa, lugares de trabajo, medidas preventivas.

Abstract

Violence in the workplace is a new problem that affects all types of sectors and professional categories. Its prevention and management requires coordinated action by all institutions involved.

According to current legislation, we need to implement preventive measures to remove or minimize this risk. To achieve this goal, we must develop working procedures establishing actions to be taken in situations of external violence. These procedures should be applied to all workers and distributed to all levels in the company.

The information and training of workers in relation to this subject is another of the preventive measures which have to be carried out, so that they acquire the necessary skills to avoid external violence. 
To know the real dimension of the problem it is necessary to record accurately all external violence situations which occur in our organization, for which it is essential to develop a notification and registration plan.

Key words: External violence, workplace, preventive measures. 


\section{INTRODUCCIÓN}

La violencia en el lugar de trabajo se ha convertido en un problema mundial; afectando a todos los sectores y categorías laborales. ${ }^{1,2}$ Es un fenómeno emergente y global, cuya prevención y manejo exige una actuación coordinada de todas las instituciones implicadas.

Muchos de los actos de violencia que tienen lugar en los centros de trabajo, no se denuncian ni registran adecuadamente, por lo que podemos afirmar que desconocemos la magnitud real del problema

Según la Organización Internacional de Trabajo (OIT), las profesiones relacionadas con el sector servicios, son las que presentan un mayor riesgo de sufrir agresiones o actos de violencia debido al estrecho contacto que se establece con los usuarios ${ }^{1,2}$.

Según la Organización Mundial de la Salud (OMS), "la violencia en el lugar de trabajo, no es un problema individual ni aislado, es un problema estructural y estratégico que tiene sus raíces en factores sociales, económicos, organizativos y culturales" por lo que debe recibir un tratamiento integral ${ }^{2}$.

La OMS destaca que dentro de la violencia en el sector servicios, la violencia en el sector sanitario es un fenómeno universal que depende de las singularidades idiosincrásicas o legislativas de cada país y que afecta por igual a los países más desarrollados y a los más pobres. Según Nordin, casi un $25 \%$ de todos los incidentes de violencia se producen en el sector sanitario y más de un $50 \%$ de los profesionales de la Sanidad han sufrido en ocasiones algún tipo de violencia. ${ }^{3}$ Sus consecuencias tienen un impacto más significativo en los países más ricos y suponen una grave amenaza tanto para la calidad y el desarrollo de la atención sanitaria como para la productividad y rentabilidad de los actuales sistemas de salud ${ }^{2}$.

La OMS advierte que la violencia en el trabajo junto al estrés representa en torno al $30 \%$ de los casos totales de enfermedades y accidentes, que afectan a los trabajadores en el sector sanitario ${ }^{2}$.

La violencia en el lugar de trabajo atenta contra la dignidad y los derechos del trabajador.

La Agencia Europea para la Seguridad y la Salud advierte que los profesionales pueden sufrir graves consecuencias dependiendo del tipo de agresión, desde la desmotivación profesional hasta el estrés y los daños físicos y psíquicos ${ }^{4,5,6}$.

La gravedad de las lesiones físicas debidas a una agresión son múltiples y de distinta consideración (contusión, heridas leves o superficiales, heridas que precisen intervención quirúrgica, heridas muy graves y mortales) ${ }^{4,5,6}$.

Respecto a las repercusiones psicológicas se ha de considerar la susceptibilidad individual del trabajador afectado, así como la intensidad, frecuencia o duración del suceso y la actuación del grupo de trabajo o de la organización; pudiendo manifestarse como ansiedad, depresión o estrés postraumático, además puede producirse abuso de medicamentos y sustancias tóxicas; con frecuencia también, se van a ver afectados el ámbito familiar y social, pudiendo, incluso, llevar al abandono de la profesión ${ }^{4,5,6}$.

En las empresas: la violencia conduce a un empeoramiento de los resultados, altera las relaciones interpersonales y el clima laboral, aumenta el absentismo, la rotación de los trabajadores, y disminuye la eficacia y la productividad. ${ }^{6,7,8}$

A nivel social se produce un aumento de la siniestralidad laboral, un empeoramiento de la calidad de los servicios prestados y la pérdida del prestigio de la entidad ${ }^{7,8}$ 


\section{NORMATIVA LEGAL}

Son las administraciones y sus autoridades competentes las encargadas de proporcionar el marco necesario para reducir y eliminar la violencia en el lugar de trabajo.

En la reunión de expertos de la OIT, celebrada en Ginebra del 8 al 15 de octubre de 2003 se determinó la creación de políticas para combatir la violencia en el lugar de trabajo, comprendiendo los siguientes puntos:

- Reducir/suprimir la violencia en el trabajo.

- Elaborar políticas y planes nacionales, autonómicos y locales sobre salud y seguridad laboral.

- Promover la participación de todas las partes implicadas.

- Revisar el derecho laboral e introducir legislación específica cuando sea necesario.

- Cumplir la legislación.

- Tratar de que, en los acuerdos nacionales, sectoriales, de trabajo y entre empresas, se incluyan disposiciones encaminadas a reducir y suprimir la violencia laboral.

- Fomentar la aplicación de políticas y planes en el lugar de trabajo, que vayan destinados a combatir la violencia laboral.

- Crear campañas de concienciación sobre los riesgos de la violencia en el trabajo.

- Registrar y hacer estudios estadísticos sobre el alcance, las causas y las consecuencias de la violencia en el lugar de trabajo.

- Coordinar los esfuerzos de las distintas partes implicadas.

El marco normativo que sustenta el Plan de Prevención y Actuación frente a situaciones de violencia externa es el siguiente:

- El Convenio sobre discriminación y empleo 1958, (núm. 111) en el que se establece que toda política o medida encaminada a combatir la violencia en el lugar de trabajo debería promover el trabajo decente y el respeto mutuo y promocionar la igualdad de trato entre hombres y mujeres.

- El Convenio sobre seguridad y salud de los trabajadores, 1981 (núm. 155), dice que un medio ambiente seguro y saludable facilita un estado de salud físico y mental óptimo en relación con el trabajo, y contribuye a prevenir la violencia en el lugar de trabajo.

- El artículo 40.2 de la Constitución Española encomienda a los poderes públicos el deber de velar por la seguridad e higiene en el trabajo.

- La Ley General de Sanidad 14/1986, en su artículo 21 establece la necesidad de "promover la salud integral del trabajador".

- La DIRECTIVA 89/391/CEE, de 12 de junio, relativa a la aplicación de medidas para promover la mejora de la seguridad y de la salud de los trabajadores en el trabajo, en su artículo 5 dice "El empresario deberá garantizar la seguridad y la salud de los trabajadores en todos los aspectos relacionados con el trabajo".

- La Ley 31/1995 de 8 de noviembre sobre Prevención de Riesgos Laborales, en el artículo 14.2, afirma que "el empresario deberá garantizar la seguridad y la salud de los trabajadores a su servicio en todos los aspectos relacionados con su trabajo".

- La Ley Orgánica 1/1992, sobre Protección de la Seguridad Ciudadana, en el artículo 13 señala que «el Ministerio de Justicia e Interior podrá ordenar, conforme a lo que se disponga reglamentariamente, la adopción de medidas de seguridad necesarias en establecimientos e instalaciones industriales, comerciales y de servicios, para prevenir la comisión de actos delictivos que puedan cometer contra ellos, cuando generen riesgos directos para terceros o sean especialmente vulnerables...» Tratando de dar respuesta a ello, están en vigor la Ley 23/1992 de Seguridad Privada, el Reglamento de Seguridad Privada (aprobado por Real 
Decreto 2364/1994) y la Orden de 23 de Abril de 1997 por la que se concretan determinados aspectos en materia de medidas de seguridad, en cumplimiento del Reglamento de Seguridad Privada.

- El Estatuto Marco del Personal Estatutario de los Servicios de Salud, aprobado mediante la Ley 55/2003 de 16 de diciembre, en su artículo 17.h) dispone que el personal sanitario ostenta el "derecho a recibir asistencia y protección de las Administraciones Públicas y Servicios de Salud en el ejercicio de su profesión o en el desempeño de sus funciones".

- Referente a los Derechos y Deberes de los ciudadanos: La Ley 41/2002 de 14 de Noviembre, Básica reguladora de la Autonomía del Paciente menciona el derecho a la información y derecho a consentir. Deber de respeto hacia los profesionales y respeto a las instituciones. Difundir que la institución sanitaria demandará al usuario que no respete las normas establecidas en cada centro o al personal que presta servicio en el mismo.

Hay comunidades autónomas que regulan estas situaciones a través de distintos procedimientos como ocurre en las Comunidades Autónomas de Castilla La Mancha, Andalucía, Asturias, Castilla León y Murcia entre otras.

La Consejería de Sanidad de la Comunidad de Madrid, reconociendo el progresivo incremento de agresiones a los profesionales sanitarios, publica la Orden 212/2004 por la que establece las directrices para la elaboración de planes de prevención y atención frente a potenciales situaciones conflictivas con los ciudadanos ${ }^{10}$.

Posteriormente ha sido modificada por la Orden 683/2006 por la que se establecen las directrices y líneas generales para la elaboración de Planes de Prevención y Atención frente a situaciones conflictivas con los ciudadanos en los centros e instituciones sanitarias y crea la Comisión Central de Seguimiento ${ }^{11}$.

En el año 2008, a través de la Orden 22/2008, de 21 de enero, se crea el fichero de datos de carácter personal denominado plan regional de prevención de situaciones conflictivas dependiente de la Dirección General de Recursos Humanos de la Consejería de Sanidad de la Comunidad de Madrid ${ }^{12}$.

Recientemente a través de la Resolución de la Dirección General de Recursos Humanos de 29 de enero de 2009, se dictan instrucciones para la puesta en marcha del registro centralizado de situaciones conflictivas ${ }^{13}$.

\section{TIPIFICACIÓN PENAL DE LAS SITUACIONES CONFLICTIVAS}

Según el artículo 10 del Código Penal "Son delitos o faltas las acciones u omisiones dolosas o imprudentes penadas por la ley".

Los Delitos y Faltas más frecuentes derivados de la violencia externa según la Ley Orgánica 10/1995, de 23 de noviembre son:

- Faltas contra las personas.

- Faltas contra el orden público.

- Delitos: Lesiones, amenazas, calumnias e injurias y atentados contra la autoridad, sus agentes y los funcionarios públicos, y de la resistencia y desobediencia.

Según el artículo 147 de la Ley Orgánica 10/1995, de 23 de noviembre se considera Delito de Lesiones. El que, por cualquier medio o procedimiento, causare a otro una lesión que menoscabe su integridad corporal o su salud física o mental, será castigado como reo del delito de lesiones con la pena de prisión de seis meses a tres años, siempre que la lesión requiera objetivamente para su sanidad, además de una primera asistencia facultativa, tratamiento médico o quirúrgico. La simple vigilancia o seguimiento facultativo del curso de la lesión no se considerará tratamiento médico. 
De todo delito o falta nace una acción penal para el castigo del culpable, y puede nacer también una acción civil para la restitución, la reparación del daño y la indemnización de perjuicios causados por el hecho punible. Excepto las lesiones y los malos tratos de obra, el resto de infracciones, cuando por su gravedad no son constitutivos de delito, sólo se pueden perseguir mediante denuncia de la persona afectada, es decir del trabajador víctima de la actuación violenta.

El asesoramiento al trabajador agredido es complejo y será el propio afectado quién decida entre las posibles acciones legales a adoptar; así como la entidad jurídica de la institución u organismo que quiere que lo represente (sindicatos, colegios profesionales, servicios jurídicos de la administración o de la entidad correspondiente).

Deberá reunirse la documentación y la información disponible respecto a la situación violenta, analizándola y completándola en caso necesario; tras la entrevista con el asesor jurídico y según la información y el material disponible se iniciará la acción jurídica elegida por el trabajador afectado.

Según el artículo 262 y siguientes de la Ley de Enjuiciamiento Criminal, recoge la obligación de denunciar todo delito o falta y establece que la denuncia podrá ser verbal o escrita y que podrá hacerse personalmente o mediante mandatario con poder especial.

La denuncia es una declaración de conocimiento consistente en un acto de información a la autoridad competente de un hecho con apariencia de delito o falta.

La denuncia que se hiciere por escrito deberá estar firmada por el denunciante.

La denuncia se interpondrá ante los Cuerpos y Fuerzas de Seguridad del Estado, Ministerio Fiscal, Tribunal competente, o Juez de Instrucción de la localidad donde ocurran los hechos denunciados.

La denuncia debe especificar los datos personales del agredido, recomendándose que como dirección a efectos de notificaciones se indique la del centro de trabajo.

Igualmente se ha de identificar al agresor indicando sus datos personales si se conocen. Si no es así, se hará una descripción del mismo y de todas las circunstancias que pudieran ayudar a identificarlo.

Se deberá recoger una relación detallada de los hechos, así como las circunstancias en que se desarrollaron los mismos (descripción del lugar, momento de la agresión, testigos, etc.).

El denunciante no tiene que probar los hechos.

Se recomienda que sea la víctima de la agresión quien presente la denuncia, a excepción de aquellos casos de lesiones dolosas en las que se prevea la posibilidad de que se repita la agresión, en cuyo caso se recomienda que sea el responsable del centro de trabajo quien la presente.

\section{VIOLENCIA EN EL ÁMBITO LABORAL: ACCIDENTE DE TRABAJO}

El impacto que un incidente violento tiene sobre la salud y la seguridad de los trabajadores varía, dependiendo de la naturaleza y gravedad del mismo, de las características del trabajador así como de la defensa que hace el individuo y la organización ante la situación.

Es importante dar una respuesta rápida y profesional que permita la recuperación del trabajador afectado por un suceso traumático. Este apoyo ha de realizarse lo antes posible, en ocasiones, es necesario que se prolongue en el tiempo, especialmente cuando las consecuencias físicas o emocionales son duraderas.

Los trabajadores afectados por la violencia en el lugar de trabajo tienen la posibilidad de someterse al tratamiento médico y psicológico necesario para recuperación de sus lesiones ${ }^{6,8,9}$. 
Pueden producirse daños físicos, siendo la gravedad de las lesiones variables: ausencia de lesión traumática visible, contusiones únicas o múltiples (hematoma, erosión, excoriación), heridas leves o superficiales que precisen aproximación (Steri-Strip ${ }^{\mathrm{TM}} \mathrm{o}$ sutura cutánea), heridas graves (que precisen sutura quirúrgica o intervención quirúrgica), heridas muy graves que conlleven riesgo vital, heridas mortales.

Las lesiones físicas suelen acompañarse de daños psíquicos, la gravedad de los mismos depende de las circunstancias de la agresión, del estado personal y del entorno de la víctima en el momento de la agresión.

- Puede producirse ansiedad que se manifiesta con miedo a seguir ejerciendo la propia profesión y, en ocasiones, verdadero pánico al lugar de trabajo, con palpitaciones, sofocos, disnea y diversas somatizaciones como gastralgias, migrañas y contracturas musculares.

- Depresión: caracterizada por una baja autoestima, que puede derivar en una sensación de incapacidad para continuar ejerciendo la profesión, dado que falta la suficiente energía e ilusión para hacer frente a las situaciones cotidianas, mostrando tristeza, apatía y falta de interés por el propio entorno.

- Estrés postraumático con episodios de insomnio y pesadillas nocturnas en las que se revive la agresión que se ha sufrido con interrupciones frecuentes del sueño que impiden la imprescindible reparación del cansancio. Suele curarse o remitir antes de un año después del incidente, pero un $30 \%$ de los casos tienden a sufrir un estrés postraumático crónico. En ocasiones puede derivar en situaciones de invalidez para el ejercicio de la profesión.

La intervención y asistencia frente a las lesiones producidas por violencia en el lugar de trabajo ha de tener las mismas características que las producidas ante cualquier accidente de trabajo ya que según el artículo 115 de la Ley General de Seguridad Social "el accidente de trabajo es toda lesión corporal que el trabajador sufra con ocasión o por consecuencia del trabajo que ejecute por cuenta ajena”.

Se efectuará el registro de la lesión como accidente de trabajo, la investigación del mismo siempre que fuere necesario, así como la planificación de las actividades preventivas resultantes.

El trabajador tiene derecho a una atención medico-sanitaria y rehabilitadora (no hay período de carencia, desde que entra a trabajar), el tratamiento se prolongara hasta la máxima rehabilitación y si es necesario se realizará una adaptación laboral y social.

\section{PROTOCOLOS DE ACTUACIÓN}

Mediante la instauración de un Protocolo específico de actuación ante situaciones de violencia en los centros de trabajo, se pretende mejorar las condiciones de salud y seguridad de los trabajadores, además de: ${ }^{4,6,8,9,14}$

- Disminuir las posibilidades de conflicto entre los usuarios y trabajadores.

- Disminuir los riesgos de agresión y el efecto lesivo de las agresiones en caso de que se produzcan.

- Prevenir agresiones y actos de violencia contra los trabajadores.

- Proteger a los trabajadores, usuarios, bienes y servicios de los centros de trabajo, mediante los medios materiales y humanos disponibles.

- Generar seguridad y confianza en los trabajadores con apoyo administrativo y jurídico.

- Mejorar la calidad de la atención prestada y generar un clima de respeto mutuo y buena comunicación. 
Según el artículo 15 de la Ley de Prevención de Riesgos Laborales, los principios preventivos: "Evitar los riesgos, evaluar los riesgos que no se puedan evitar, combatir los riesgos en su origen, adaptar el trabajo a la persona, tener en cuenta la evolución de la técnica, sustituir lo peligroso por lo que entrañe poco o ningún peligro, planificar la actividad preventiva, aplicar protección colectiva e individual", son la base para evitar situaciones de riesgo.

La aplicación de estos principios preventivos son prioritarios, con el fin de evitar el riesgo y si ello no fuera posible adoptar las medidas necesarias para minimizarlo, que irán dirigidas a la reducción o eliminación de la incidencia y gravedad de los mismos.

Para poder poner en marcha las medidas preventivas adecuadas, debemos conocer las causas que han originado las conductas violentas y agresivas ${ }^{15}$ :

Existen muchas teorías sobre las causas de la violencia. Algunas destacan que los comportamientos agresivos son fruto del aprendizaje, bien por imitación de acontecimientos familiares similares, culturales, de los propios medios de comunicación (Bandura) o por la posible ausencia de consecuencias que sigue, en ciertos casos, a un cierto acto de violencia, lo que tiende a reforzar la conducta violenta (Berkowitz).

También puede explicarse el incremento de las tasas de violencia por la adopción de valores que soportan la violencia (Wolfgang y Ferracuti).

Como vemos las causas de la violencia pueden ser muy variadas, unas son fácilmente identificables; pero en ocasiones, la violencia sigue un curso gradual, se inicia por situaciones leves y se va pasando a niveles de mayor intensidad. La propia reacción de los trabajadores ante tal situación puede contribuir a determinar el curso de los acontecimientos en un sentido u otro.

Lo que resulta importante, como punto de partida, es proceder al análisis de las situaciones potencialmente agresivas que se producen en los centros de trabajo para identificar los riesgos $1,2,4,6,8$.

Los factores de riesgo pueden estar relacionados con el lugar de trabajo, los trabajadores y el agresor.

Los factores de riesgo relacionados con el lugar de trabajo pueden ser debidos a:

- Falta de información institucional, por parte del personal, que satisfaga las preguntas y preocupaciones del usuario.

- Insuficiencia de recursos humanos y materiales (plantillas inadecuadas).

- Sobrecarga de trabajo de los profesionales.

- Descoordinación entre el personal y los servicios del centro, que puede originar información contradictoria al ciudadano.

- Trabajo en solitario o grupos reducidos.

- Interrupción en las consultas con la visita ya iniciada.

- Demora en la asistencia y largas esperas.

- Defectos en la información y atención prestada al usuario.

- Estilos de gestión rígidos y autoritarios.

- Trabajo nocturno y a turnos.

- Salas de espera y lugares comunes inadecuados.

- Zonas deprimidas o con alto índice de criminalidad.

- Trabajo con público (consumidores, clientes, pasajeros, pacientes).

- Trabajo que implica intercambio regular de dinero con clientes.

Los factores de riesgo relacionados con el trabajador pueden ser debidos a:

- Falta de habilidad de comunicación con los usuarios.

- Interferencia de los problemas personales en el trabajo. 
- Insatisfacción profesional.

- Falta de empatía con el usuario o cliente.

- Desconocimiento de la organización en la que se trabaja o de los circuitos complementarios.

- Descoordinación con otros profesionales para orientar adecuadamente al usuario.

- Negativas no razonadas a las peticiones efectuadas por el usuario.

- Situaciones de mobbing o burnout.

- Temperamento o carácter difícil del trabajador.

- Demora, errores, incumplimientos en las peticiones del cliente.

Los factores de riesgo relacionados con el usuario pueden ser debidos a:

- Ciudadanos con rasgos de personalidad en los que destaca un control de impulsos deficitario, baja resistencia a la frustración o cierto perfil de agresividad (lenguaje con tono alto, insultos, palabras groseras).

- Ciudadanos que persiguen un beneficio personal fraudulento y para conseguir sus objetivos presionan, amenazan y agreden al profesional.

- Clientes poco receptivos a las explicaciones y que no atienden a los motivos que se les indica.

- Individuos que no admiten y que a menudo infringen las normas de conducta social establecida. Reaccionan agresivamente ante cualquier contrariedad.

- Ciudadanos cuyas expectativas en torno a la atención que demandan son superiores o incluso distintas a las recibidas.

- Experiencias previas negativas con la atención solicitada.

- Individuos con inestabilidad psicológica.

\section{PLAN DE PREVENCIÓN FRENTE A VIOLENCIA EXTERNA}

El plan de prevención ante violencia externa contempla:

- Prevención de potenciales situaciones de violencia externa.

- Actuación ante situaciones de violencia externa.

- Asesoramiento y apoyo al trabajador.

\section{Prevención de potenciales situaciones de violencia externa}

1. Reforzar las garantías en la prestación de los servicios realizados.

2. Reforzar la seguridad para la prevención secundaria de las agresiones.

3. Impulsar la formación de los trabajadores en el manejo de situaciones de violencia externa ${ }^{4,6}$.

\section{Reforzar las garantías en la prestación de los Servicios realizados}

- Informar a los ciudadanos de las prestaciones de los servicios solicitados:

- Conocerán sus derechos y deberes.

- Mejorar la accesibilidad y la atención solicitada:

- Revisar la atención telefónica.

- Adecuar plantillas de personal administrativo.

- Disminuir los tiempos de espera.

- Asegurar los derechos de los usuarios:

- Establecer procedimientos de emergencia claros en caso de incidente. 
- Rotar puestos o zonas de trabajo de alto riesgo de forma que la misma persona no esté siempre sujeta al mismo riesgo.

- Colocar los artículos de valor fuera del alcance de los clientes.

- Colocar mostradores anchos o elevar la altura de los mostradores.

- Asegurarse que los trabajadores tienen un acceso rápido a zonas seguras.

- Cambiar la disposición de las zonas de espera, haciendo de éstas zonas confortables.

- Asegurar una buena iluminación exterior e interior para evitar zonas de cobertura a los asaltantes.

- Instalar pantallas o sistemas de protección en áreas donde los trabajadores tengan más riesgo.

- Monitorizar las entradas, salidas y las zonas de mayor riesgo.

\section{Reforzar la seguridad para la prevención secundaria de la violencia externa}

- Seguridad Pasiva:

- Dispositivos de alarma (teléfono, interfono, timbres).

- Videocámaras: grabación espacios comunes.

- Seguridad Activa:

- Comunicación con las Fuerzas de Seguridad del Estado.

- Confidencialidad de los datos personales de los trabajadores.

- Acompañamiento de otro profesional.

- Protocolos con empresa de seguridad, en ruta o permanente.

\section{Impulsar la formación de los trabajadores en el manejo de situaciones de violencia externa}

La Ley de Prevención de Riesgos Laborales, en su artículo 19 bajo el título "Formación de los trabajadores" establece "la obligación del empresario de garantizar que los trabajadores recibirán una formación teórica y práctica, suficiente y adecuada..." centrada específicamente en su puesto de trabajo. Por ello:

- Se fomentará la formación del personal en el manejo de violencia externa.

- Se facilitará y organizará la realización de dichos cursos.

En dicha formación se hará referencia a:

- Principios básicos de la comunicación en situaciones difíciles.

- Elementos que facilitan y dificultan la comunicación.

- La asertividad: diferentes técnicas para mejorar la asertividad.

- La escucha activa.

- La comunicación no verbal.

- Técnicas y habilidades de negociación.

- Autocontrol emocional y manejo del estrés.

- Ayudar a reconocer y entender los condicionantes personales, sociales, ambientales y psicológicos que generan agresividad.

- Analizar los diversos tipos y niveles de la agresividad.

Las Recomendaciones o Consejos generales de actuación que se darán al personal ante situaciones de violencia externa son las siguientes:

- Intentar mantener una escucha activa y relajada.

- No responder a las agresiones verbales, no insultar, no desafiar al agresor. 
- Tomar precauciones, evitar tener objetos corto-punzantes o contundentes a mano del presunto agresor.

- Cuando fuese preciso, interrumpir la actividad hasta que la situación esté controlada, reanudándola cuando se garantice la atención al usuario.

- En usuarios con antecedentes de conflictividad con un determinado profesional, se procurará el cambio del mismo.

Recomendaciones para el manejo del ciudadano agresivo:

- Mantener el equilibrio emocional y no responder a las provocaciones.

- Conversar con corrección, en un tono afable y profesional.

Si aumenta la tensión del usuario reorientar la conversación:

- Hacer contrabalance emocional.

- Liberar la tensión emocional del usuario.

- Avisar a un compañero para que se integre.

- Reconocer las posibles deficiencias de la organización.

- Manifestar los propios sentimientos al usuario.

Durante la entrevista es aconsejable evitar las siguientes señales verbales y no verbales:

Verbales:

- Evitar comunicación que genere hostilidad.

- Evitar verbalizar amenazas, coacciones o insultos.

- Evitar minimizar la situación, las quejas y críticas.

- Evitar el rechazo de peticiones.

- Evitar transmitir información errónea o que no se ajuste a la realidad.

No verbales:

- Evitar lenguaje y posturas desafiantes.

- Evitar el contacto físico y el exceso de proximidad.

- No invadir su espacio personal.

- Evitar el contacto ocular prolongado.

- Evitar los movimientos rápidos y dar la espalda.

Si a pesar de todo, ha sido imposible reconducir la situación y esta persiste se preparará la salida del lugar y en caso necesario se solicitará la intervención de los cuerpos de seguridad.

\section{Actuación ante situaciones de violencia externa}

Cuando se ha generado el conflicto y en función del tamaño de la organización y de la actividad realizada, la dirección deberá establecer un sistema de gestión de la violencia en el lugar de trabajo, que incluya:

- Una política de seguridad y salud relativa a la violencia en el lugar de trabajo; que deberá acompañarse de iniciativas encaminadas a sensibilizar a empresarios, trabajadores, usuarios y público en general.

- Implicación de todos los estamentos y difusión a todos los trabajadores de la organización.

- Pautas de actuación de los actores implicados.

- Un sistema de registro preciso que recoja las características de la violencia en el lugar de trabajo, la categoría de los actos violentos y la repercusión que han 
tenido en el entorno laboral. Es importante documentar todos los actos de violencia para que cada organización aprenda de sus propias experiencias.

- Disposiciones, procedimientos, instrucciones y otros documentos internos, redactados con claridad y presentados de manera comprensible para quienes deban utilizarlos.

- Medidas de intervención y control de los incidentes violentos.

- Acceso razonable a los registros sobre la violencia en el lugar de trabajo, respetando siempre la confidencialidad.

- Elaboración de mapas de riesgos.

- Apoyo médico y psicológico al trabajador afectado.

Siguiendo las recomendaciones prácticas sobre la violencia en el lugar de trabajo en el sector servicios y las medidas para combatirla (OIT) ${ }^{1,4}$, se creará un grupo de trabajo con el fin de identificar y evaluar los riesgos o amenazas potenciales y definir una serie de intervenciones para disminuirlas:

- Investigación y análisis de los datos disponibles sobre incidentes violentos.

- Evaluación y comprobación periódica del funcionamiento y resultados de los:

- Sistemas de control y vigilancia de los programas y medidas de seguridad.

- Sistemas de información y formación en materia de seguridad laboral a los trabajadores.

- Sistemas de asesoramiento y soporte a la víctima de una agresión.

- Sistemas de divulgación de los incidentes violentos.

- Sistemas de participación de los trabajadores en la preparación de los criterios para evaluar, prevenir y gestionar los riesgos de violencia en el propio lugar de trabajo.

- Información, asesoramiento y soporte, para gestionar la situación inmediata.

- Tratamiento de las consecuencias urgentes o inmediatas de la agresión al trabajador

Una vez que ha ocurrido el incidente se inicia un proceso de investigación y análisis de lo acontecido, dirigido a:

- Recopilar información y documentación del incidente.

- Declarar incidentes violentos por parte de los trabajadores afectados.

- Analizar e identificar la información en relación con los siguientes aspectos:

- Los peligros más inminentes y graves.

- Los defectos en el funcionamiento.

- Las influencias ambientales negativas (diseño, iluminación, visibilidad, mobiliario, accesos, medidas de seguridad del lugar del trabajo, etc.).

- Los déficit del personal y de la organización.

- Analizar los factores del entorno de trabajo: localización geográfica del centro de trabajo, características socioeconómicas del entorno, etc.

- Analizar los procedimientos de trabajo que puedan entrañar mayor riesgo: gestión de dinero con el público, gestión de caja, gestión de esperas y colas, gestión de quejas y reclamaciones, cantidad y ritmo de trabajo y asistencia sanitaria.

Identificado el riesgo, hay que evaluar la probabilidad de ocurrencia y la gravedad de los daños para efectuar una planificación de medidas preventivas, definir el orden de prioridades para abordar los riesgos de violencia en el lugar de trabajo y a continuación indicar las medidas de prevención y protección que es preciso adoptar frente al riesgo. Se debe recomendar un seguimiento de la situación hasta su resolución. 


\section{Apoyo al trabajador}

- Asistencia sanitaria y tratamiento médico y psicológico, rápido o urgente, inmediatamente después de producirse la agresión.

- Documentación de las lesiones a través del parte de lesiones.

- Tramitación de baja laboral por incapacidad temporal derivada de accidente de trabajo, si se precisa.

- Informar, asesorar y apoyar al trabajador agredido para gestionar la situación inmediata y en el futuro, sobre las ayudas a las que puede acceder y en relación con las diferentes acciones que es factible emprender ante un incidente violento y/o contra el autor de la agresión.

- Dar información y apoyo a la familia de la víctima de la agresión, si es necesario.

- Gestionar las compensaciones adecuadas al agredido en el seno de los instrumentos transaccionales (tribunales arbitrales, acuerdos particulares...).

\section{ACTUACIÓN ANTE VIOLENCIA EXTERNA EN EL SECTOR SANITARIO}

Según $\operatorname{Nordin}^{3}$, la violencia en el lugar de trabajo afecta especialmente a los trabajadores de la salud (el 25\% de todos los incidentes violentos se registran en este sector).

En muchos países europeos se ha optado por la vía legislativa para afrontar estas situaciones, debido al número y gravedad de los $\operatorname{casos}^{1,9}$.

\section{Datos mundiales relacionados con la violencia externa en el sector sanitario:}

Estados Unidos (EEUU), fueron los primeros en afrontar el fenómeno de la violencia en el lugar de trabajo, aquí la violencia laboral tiene una gravedad añadida por tratarse de una sociedad en la que está legalizada la tenencia de armas. Es importante destacar que el personal sanitario de EE UU afronta 16 veces más riesgo de sufrir violencia en su lugar de trabajo que cualquier trabajador de otro sector.

El National Occupational Health an Safety Administration (OSHA) es la responsable de la edición de una de las primeras guías específicas para abordar la violencia en el sector sanitario.

Canadá, Australia y Nueva Zelanda utilizaron la experiencia de EE UU para adoptar las medidas más adecuadas frente a la violencia externa en las organizaciones de trabajo, teniendo en cuenta su propia realidad.

Gran Bretaña ha visto como la violencia contra el personal del National Health Service (NHS) se convertía en una realidad alarmante, por este motivo el gobierno británico puso en marcha la campaña "Tolerancia Cero a la Violencia en el NHS".

Al margen de la experiencia británica, en Europa destaca la experiencia de Suecia, pionera en legislar en esta materia desde el año 1993. También tenemos las experiencias de Bélgica, Holanda o Italia pero hay que citar a Francia de forma especial, tanto por el número como por la gravedad de los incidentes violentos que han experimentado sus profesionales sanitarios.

\section{Datos en España en relación con la violencia externa en el sector sanitario:}

A principios del año 2003, el Colegio Oficial de Médicos de Barcelona (COMB) ${ }^{14}$ puso en marcha un programa de prevención de la violencia en él lugar de trabajo que preveía la creación del Observatorio de la Seguridad de los Médicos, se contrato una póliza de defensa jurídica para los médicos agredidos en el cumplimiento de su ejercicio profesional, se elaboro un programa de apoyo a las víctimas y se adquirió el compromiso institucional de difundir una nueva cultura de dignidad y seguridad de estos profesionales en la prestación de un acto médico. 
Hay comunidades autónomas que regulan estas actuaciones a través de distintos procedimientos, es el caso de las Comunidades Autónomas de Castilla La Mancha, "Plan director de seguridad corporativa y de protección del patrimonio del SESCAM "Plan PERSEO" ${ }^{16}$ "Plan de prevención de agresiones a los profesionales de la sanidad de la Región de Murcia" ${ }^{17}$; "Plan integral de seguridad de la gerencia regional de salud de Castilla y León" 18 entre otras.

La Consejería de Sanidad de la Comunidad de Madrid, reconociendo el progresivo incremento de agresiones a los profesionales sanitarios, publica la Orden 212/2004 ${ }^{10}$ por la que establece las directrices para la elaboración de planes de prevención y atención frente a potenciales situaciones conflictivas con los ciudadanos.

Analizando los factores de riesgo en este sector existen una serie de peculiaridades específicas que agravan la situación de conflicto generada por los usuarios, como pueden ser:

- Demora en la asistencia, pruebas e intervenciones.

- Defectos en la información y atención en situaciones de riesgo vital o emergencia (Unidad de cuidados intensivos, Urgencias).

- Elevada presión asistencial.

- Falta de empatía con el enfermo, familiares y acompañantes.

- Negativas no razonadas a peticiones de prestaciones sanitarias.

- Características especiales en pacientes difíciles: pasivo dependiente, emotivo seductor, masoquista, somatizador, exigente agresivo, incumplidor del tratamiento ${ }^{15}$.

- Ciudadanos que persiguen un beneficio personal fraudulento a través de la asistencia sanitaria (recetas, bajas) y para conseguir sus objetivos presionan, amenazan y agreden al profesional.

- Tipo de patología que presenta (gravedad de la enfermedad o complicación de la misma).

- Experiencias previas negativas con el sistema sanitario.

Es importante tener en cuenta la consideración o el paradigma de la presunta peligrosidad del paciente psiquiátrico y el paciente drogodependiente; por lo que pueden surgir actitudes negativas hacia este tipo de paciente por parte del personal asistencial, siendo fundamental la formación de los trabajadores en el manejo de este tipo de pacientes ${ }^{19}$.

Entre las Medidas Preventivas destacamos, dentro del sector sanitario, las siguientes:

\section{Reforzar las garantías en la prestación de los Servicios Sanitarios}

- Disminuir los tiempos de espera.

- Adecuar los espacios de los centros sanitarios.

- Facilitar el ingreso y la estancia de los pacientes en los centros sanitarios.

- Asegurar los derechos sanitarios y de información de los pacientes.

- Rediseño de las consultas: colocación de mobiliario de forma que, en la medida de lo posible, impida la acción agresora, así como facilitar la posible huida del agredido.

\section{Reforzar la seguridad para la prevención secundaria de la violencia externa}

\section{Atención domiciliaria:}

- Establecer acuerdos y procedimientos de actuación con las Fuerzas de Seguridad del Estado.

- Teléfono móvil institucional: telepresencia o buscapersonas.

- En caso de antecedentes de comportamiento inadecuado o enfermos conflictivos la asistencia se efectuará por dos profesionales sanitarios. 
- Si el origen de la agresión es un paciente con enfermedad mental, hay que tener en cuenta que el médico puede ordenar el internamiento del paciente según el artículo 763 de la Ley de Enjuiciamiento Civil (Ley 1/2000).

Los estudios realizados en nuestro país en relación con este tema son los siguientes:

El trabajo, becado por la Fundación Prevent durante el ejercicio $2006^{20}$, expone el diseño y los resultados de la implementación en diversos centros sanitarios de Cataluña a través de la web "www.violenciaocupacional.net", de un sistema informatizado de notificación on-line de Incidentes Violentos.

La información sobre la violencia ocupacional en el sector de la salud se obtenía básicamente de encuestas de autoinforme, con bajos porcentajes de respuesta y notables problemas metodológicos de validez, por los diversos sesgos que conlleva. Este proyecto tiene como objetivo diseñar planes de mejora, tanto en la prevención primaria como en las actuaciones post incidente. Se describe la tipología y la prevalencia de esta violencia en el colectivo investigado, identificando los principales factores de riesgo de la misma y se propone medidas para su prevención.

Informe realizado por la Universidad de Zaragoza $^{21}$, publicado con el título "Aggression towards Health Care Workers in Spain: A Multi-facility Study to Evaluate the Distribution of Growing Violence among Professionals, Health Facilities and Departments (Gascón, S: Martínez-Jarreta, B; Santed, M.A: González Andrade, J.F. y Casolod, Y) International Journal of Occupational and Environmental Health (Volumen 15, n. ${ }^{\circ} 1$ [2009]

El Consejo General de Enfermería de España ${ }^{22}$ lleva a cabo periódicamente estudios de opinión entre los enfermeros colegiados para conocer la situación de la profesión y su opinión acerca de diferentes temas de actualidad, entre estos se ha realizado un estudio sobre situaciones conflictivas.

Para llevar a cabo este estudio se han consultado un total 600 profesionales de enfermería de toda España con un nivel de error $+/-4,07 \%$ y un nivel de confianza del $95,5 \%$, y se ha observado que un $33 \%$ de estos profesionales ha sufrido una agresión física o verbal en los últimos 12 meses. De este $33 \%$, un $3,7 \%$ han sido agredidos físicamente lo que supone un total de 2.928 profesionales de enfermería agredidos en el último año.

En un estudio realizado por el sindicato SATSE a través de su portal de Internet, se refleja que 8 de cada 10 profesionales de enfermería han sido agredidos física o verbalmente en sus centros de trabajo. De ellos, el 62,64\% afirma haber sido agredido verbalmente, un $34,45 \%$ han sido agredidos tanto física como verbalmente y un $2,8 \%$ solo físicamente.

Hay un estudio realizado en los trabajadores del SERMAS (Servicio Madrileño de Salud) del área 6 de la Comunidad de Madrid, durante el año 2009 y el 2010 para determinar la incidencia de la comunicación de situaciones conflictivas con los ciudadanos, identificar el ámbito de actuación, la categoría profesional más afectada, conocer la prevalencia del tipo de agresión e identificar las consecuencias tras las situaciones conflictivas notificadas ${ }^{23}$.

\section{MODELO DE PROCEDIMIENTO DE ACTUACIÓN ANTE VIOLENCIA EXTERNA}

\section{Es un modelo esquemático que cada organización podrá adaptar a sus necesidades}

\section{Objetivo}

Este procedimiento tiene por objeto definir las actuaciones que deben llevar a cabo el trabajador, su mando y el resto de instancias implicadas, ante un incidente de violencia externa en el que medie cualquier tipo de agresión física o psíquica. 


\section{Alcance}

Todos los trabajadores de la organización en la que prestan sus servicios.

\section{Normativa aplicable}

- El artículo 40.2 de la Constitución Española encomienda a los poderes públicos el deber de velar por la seguridad e higiene en el trabajo.

- La Ley General de Sanidad 14/1986, en su artículo 21 "promover la salud integral de trabajador".

- La Directiva 89/391/CEE, de 12 de junio.

- Ley General de la Seguridad Social.

- Ley 31/95 de Prevención de Riesgos Laborales.

Además de la normativa citada, justifica la realización del procedimiento el gran alcance de este tipo de incidentes en determinados centros de trabajo, y la necesidad de sistematizar la notificación y recogida de datos al respecto, para abordar adecuadamente la evaluación del fenómeno y el establecimiento de medidas preventivas eficaces, así como la información al trabajador de las actuaciones que se deben poner en marcha ante estos incidentes.

\section{Responsabilidades}

\section{Empresario:}

Establecer una política de seguridad y salud relativa a la violencia en el lugar de trabajo; que deberá acompañarse de iniciativas encaminadas a sensibilizar a directivos, trabajadores, usuarios y público en general.

Difusión de la política en todos los estamentos de la organización.

\section{Mando intermedio:}

El Mando del trabajador que ha sufrido una situación de violencia externa, será el responsable de poner en marcha las medidas pertinentes para proteger al trabajador y garantizar la atención a los usuarios, así como notificar al departamento designado por el empresario lo acontecido.

\section{Servicio de Prevención:}

Prestará al trabajador la atención médica necesaria, realizando además la investigación y el registro del incidente, a fin de confeccionar el mapa de riesgos y establecer las medidas preventivas adecuadas.

\section{Delegados de Prevención:}

Como miembros del Comité de Seguridad y Salud conocerán la información relacionada con las situaciones de violencia externa que afecten a los trabajadores.

\section{Trabajadores:}

Serán responsables de notificar todos los incidentes que consideren de violencia externa, según el modelo existente en la organización.

\section{Grupo de Trabajo:}

Supervisará el desarrollo y puesta en marcha de dicho procedimiento. 


\section{ACTUACIONES DE LOS TRABAJADORES IMPLICADOS}

\section{Trabajador afectado:}

- Avisar al Servicio de Seguridad lo antes posible a través de la centralita telefónica o pulsando, en los casos en los que se disponga de ellos, los timbres antipánico.

- Avisar a su mando inmediato, que desde ese momento deberá tomar parte activa en el proceso.

- Notificar según modelo elaborado por la organización cualquier situación que el trabajador considere de violencia externa.

- Solicitar atención médica y parte de lesiones, si existe lesión física o psíquica.

- Notificar al Servicio de Prevención, Recursos Humanos o departamento designado por el empresario, cuando de la situación de violencia derive un Accidente de Trabajo.

\section{Servicio de Prevención:}

- Recopilar información y documentación del incidente.

- Identificar el riesgo, investigación del incidente y efectuar una planificación de medidas preventivas a adoptar.

- Seguimiento de los incidentes, explotación de datos para la elaboración del mapa de riesgos y la adopción de medidas preventivas específicas.

- Registro en la base de datos de accidentes de trabajo, en caso de que el incidente se reconozca como accidente de trabajo.

- Atención médica y seguimiento en los casos en que esté indicado.

- Formación a los trabajadores en el manejo de situaciones de violencia externa.

\section{Dirección:}

- Ofrecer recursos organizativos (recursos humanos y materiales necesarios).

- Impulsar la prevención y llevar a cabo las medidas preventivas indicadas.

- Valorar apoyo legal a los trabajadores afectados.

- Valorar procedimientos disciplinarios y de reclamación.

\section{INFORMACIÓN, SEGUIMIENTO Y CONTROL}

El Comité de Seguridad y Salud será informado periódicamente por el Servicio de Prevención de los incidentes que se produzcan y, puntualmente, en caso de que el incidente sea calificado de grave, bien por los daños personales o materiales ocasionados, o por el número de trabajadores afectados.

Se establecerá la explotación periódica de los datos, sometiéndose a seguimiento por el grupo de trabajo a fin de analizar el mapa de riesgos y establecer las medidas preventivas pertinentes.

\section{DIVULGACIÓN DEL PROCEDIMIENTO}

El Procedimiento general de actuación ante violencia externa, deberá ser divulgado entre todos los trabajadores, fundamentalmente entre los mandos intermedios, que deben conocer el circuito e implicarse en el proceso. 


\section{REGISTRO Y ARCHIVO}

El Servicio de Prevención registrará y archivará toda la documentación generada en la tramitación de las situaciones de violencia externa que hayan sido notificadas por los trabajadores.

\section{MODELO DE REGISTRO DE SITUACIONES DE VIOLENCIA EXTERNA}

Datos que deben ser recogidos en el modelo de notificación:

- Datos del trabajador afectado:

- Datos personales.

- Categoría laboral.

- Departamento o servicio al que pertenece.

- Datos referentes al incidente:

- Lugar, día y hora del incidente.

- Tipo de incidente, descripción de los hechos.

- Probable causa desencadenante.

- Presencia o no de testigos.

- Consecuencias del incidente:

- Lesión física.

- Lesión psíquica.

- Suspensión de la actividad.

- Baja laboral.

- Si ha precisado atención médica y/o atención psicológica.

- Actuaciones posteriores al incidente:

- Si ha sido necesaria la intervención de otras personas (compañeros de trabajo, seguridad privada, fuerzas de seguridad del estado...).

- Ayuda por parte de la empresa:

- Si ha necesitado ayuda jurídica, asesoramiento y /o apoyo organizativo, etc.

- Datos referentes al agresor:

- Número de agresores.

- Edad aproximada.

- Genero.

- Tipo de agresor (cliente, acompañante).

- Si es o no reincidente.

- Seguimiento del incidente.

- Fecha de notificación del incidente.

— Firma del trabajador que ha sufrido el incidente.

\section{CONCLUSIONES}

- Implicación por parte de todas las instituciones, dedicando los recursos humanos y materiales necesarios para eliminar o disminuir estas situaciones de violencia externa.

- Elaboración de un procedimiento de actuación en el que estén implicados todos los estamentos de la empresa. 
- Difusión adecuada del procedimiento en cada uno de los centros de trabajo. Todos los trabajadores deben conocer el procedimiento de actuación ante violencia externa

- Sensibilizar a los trabajadores para la notificación de las situaciones de violencia externa que se produzcan en los centros de trabajo

- Establecer medidas preventivas dirigidas a disminuir los casos de violencia externa, entre otras, mejorar la información a los usuarios y aumentar la formación de los trabajadores para asegurar la correcta actuación de los mismos ante este tipo de situaciones

\section{REFERENCIAS BIBLIOGRÁFICAS}

1. OIT, OMS, CIE, ISP: Directrices Marco para afrontar la violencia laboral en el sector de la salud. Ginebra 2002.

2. OIT, OMS, CIE, ISP.Ops. Cit.

3. Nordin, H. Sistema de información sobre las lesiones en el trabajo. 1995.

4. Agencia Europea para la Seguridad y la Salud en el Trabajo. F. 24, La violencia en el trabajo. 2002.

5. Kristensen TS. Job stress and cardiovascular disease: a theoretic critical review. J Occupy Health Psycho 1996.

6. Luna, M. Riesgos Psicosociales en los empleados de la Administración Pública en la Comunidad de Madrid. USMR. CCOO 2006.

7. Yela, C. Relación de los profesionales sanitarios y los pacientes en la violencia en el sector sanitario.

8. Di Martino, V. Violence at work. Ginebra: Internacional Labour Organization. 1998.

9. NTP 489-1998. Violencia en el lugar de trabajo. INSHT.

10. Orden 212/2004, de 4 de marzo, del consejero de Sanidad y Consumo, por las que se establecen las directrices y líneas generales para la elaboración de planes de prevención y atención frente a potenciales situaciones conflictivas con los ciudadanos en los centros e instituciones sanitarias públicas.

11. Orden $683 / 2006$, de 24 de marzo de la Consejería de Sanidad y Consumo, por la que se modifica la Orden 212/2004 de 4 de marzo de la Consejería de Sanidad.

12. Orden $22 / 2008$, de 21 de enero, se crea el fichero de datos de carácter personal denominado plan regional de Prevención de situaciones conflictivas dependiente de la Dirección General de RRHH de la Consejería de Sanidad de la Comunidad de Madrid.

13. Resolución de la Dirección General de Recursos Humanos de 29 de enero de 2009, se dictan instrucciones para la puesta en marcha del Registro Centralizado de la Consejería de Sanidad para Situaciones conflictivas con los ciudadanos.

14. Quaderns de la bona praxi. Guia per preveni i gestionar la violència en el lloc de treball contra els metges. Col.legi Oficial de Metges de Barcelona. 2004.

15. El paciente difícil: quién es y cómo manejarlo J. Ágreda, E. Yanguas Anales suplemento 2.

16. Plan director de seguridad corporativa y de protección del patrimonio del SESCAM. Plan Perseo

17. Plan de prevención de las agresiones de los profesionales de la sanidad de la Región de Murcia.

18. Plan integral de seguridad de la gerencia regional de Salud de la Junta de Castilla y León.

19. Conflictos y dilemas éticos en la atención psiquiátrica Santander F, Hernanz M, Cuadernos de psiquiatría comunitaria, vol 1, número 2 pp 122-139, 2001.

20. Violencia hacia el profesional de la salud: prevención, manejo y actuación post incidente. Creación de un registro de notificación por Internet en Cataluña. Genís Cervantes i Ortega.

21. Aggression Towards Health Care Workers in Spain: A Multi-facility Study to Evaluate the Distribution of Growing Violence Among Professionals, Health Facilities and Departments (Gascón, S: Martínez-Jarreta, B; Santed, M.A: González Andrade, J.F. y Casolod, Y) International Journal of Occupational and Environmental Health (Volúmen 15, n. ${ }^{\circ} 1$ [2009]).

22. Estudio sobre agresiones a la profesión enfermera. Organización Colegial de Enfermería Consejo General.

23. Incidencia de notificación de situaciones conflictivas con los ciudadanos en el Área 6 de la Comunidad Autónoma de Madrid. Maestre Naranjo, M, Borda. J, Revista Medicina y Seguridad. Med. segur. trab. vol.58 $\mathrm{n}^{\circ} .223$ Madrid Junio 2011. 